\title{
96. Relations between Measure and Topology in some Boolean Space.
}

\author{
By Yoshimiti MiBU. \\ Mathematical Institute, Nagoya Imperial University. \\ (Comm. by T. TAKAGI, M.I.A., July 12, 1944.)
}

Let $\Omega$ be a bicompact Hausdorff space the closure of whose open set is open. We assume that the class $\mathbb{E}$ of all the closed-open sets constitutes the base of $\Omega$. $\&$ is a finitely additive class which contains $\Omega$ and the empty set $\mathfrak{D}$. Let there be defined on $\&$ a Jordan measure $m(E)$ with the following two conditions:

$1 m(\Omega)=1, m(E)=0$ if and only if $E=\mathfrak{D}$.

$2 \lim _{n \rightarrow \infty} m\left(E_{n}\right)=m\left(\left(\bigcup_{n=1}^{\infty} E_{n}\right)^{\alpha}\right)$ for any ascending sequence $\left\{E_{n}\right\}$ of sets $\epsilon\left(\mathfrak{S}^{1)}\right.$.

The purpose of the present note is to discuss the relations between measure and topology in $\Omega$. Our main result is resumed in the theorems 10, 11 and 13 below.

Theorem 1. We have

$$
\sum_{n=1}^{\infty} m\left(E_{n}\right) \geqq m\left(\left(\bigcup_{n=1}^{\infty} E_{n}\right)^{a}\right)
$$

for every sequence $\left\{E_{n}\right\}$ of sets $€ \mathbb{E}$, and the equality holds good if and only if $E_{n}$ are mutually disjoint. In particular, we have

$$
\sum_{n=1}^{\infty} m\left(E_{n}\right)=m\left(\bigcup_{n=1}^{\infty} E_{n}\right)
$$

if $\bigcup_{n=1}^{\infty} E_{n} \in \&$. Thus the Jordan measure $m(E)$ is countably additive on $C$.

Definition 1. (of outer measure $m^{*}$ ). For any set $A \leqq \Omega, m^{*}(A)$ denotes the infimum of $m(E)$ where $E \in ほ, E \geqq A$

Theorem 2.

$$
\begin{gathered}
m^{*}(A) \leqq m^{*}(B) \text { if } A \leqq B \\
m^{*}(A)=m(A) \text { if } A \in \mathbb{E} \\
m^{*}(A+B) \leqq m^{*}(A)+m^{*}(B) \\
m^{*}(A)=m^{*}\left(A^{a}\right)
\end{gathered}
$$

Definition 2. (of inner measure $m_{*}$ ). For any set $A \leqq \Omega, m_{*}(A)$ denotes the supremum of $m(E)$ where $E \in[, E \leqq A$.

Theorem 3. of $A$.

1) $A^{a}, A^{c}$ and $A^{i}$ respectively denote the closure, the complement and the interior 
No. 7.J Relations between Measure and Topology in some Boolean Space.

$$
\begin{gathered}
m_{*}(A) \leqq m_{*}(B) \quad \text { if } \quad A \leqq B \\
m_{*}(A)=m(A) \quad \text { if } \quad A \in \mathbb{E} \\
m_{*}(A)=m_{*}\left(A^{i}\right) \\
m^{*}(A) \geqq m_{*}(A) . \\
m^{*}(A)+m_{*}(A)=1 .
\end{gathered}
$$

Theorem 4.

Lemma 1.

Proof. For every $\varepsilon>0$, there exists a set $E € \leftleftarrows$ such that $E \geqq A$ and $m^{*}(A)+\varepsilon>m(E) . \quad E$ and $E^{c}$ both belongs to $\mathbb{E}$ and thus we have $m(E)+m\left(E^{c}\right)=m(\Omega)=1$. We have $m_{*}\left(A^{c}\right) \geqq m\left(E^{c}\right)$ from $E^{c} \leqq A^{c}$ and thus $m^{*}(A)+\varepsilon>m(E)=1-m\left(E^{c}\right) \geqq 1-m_{*}\left(A^{c}\right)$. Therefore $m^{*}(A)$ $+m_{*}\left(A^{c}\right) \geqq 1$.

Similarly, there exists, for every $\varepsilon>0$, a set $E \in(\xi$ such that $E \leqq A^{c}$ and $m_{*}(A)-\varepsilon<m(E)$. From this we obtain $m_{*}(A)-\varepsilon<m(E)$ $=1-m\left(E^{c}\right) \leqq 1-m^{*}(A)$ as above, and so

$$
m^{*}(A)+m_{*}(A) \leqq 1 \quad \text { Q. E. D. }
$$

Lemma 2. For any set $A$ there exists an open set $H \leqq A$ such that $m_{*}(A)=m\left(H^{a}\right)$ and $A-H$ does not contain open set $\neq \mathfrak{D}$.

Proof. From the definition of $m_{*}(A)$, there exists a sequence $\left\{E_{n}\right\}$ of sets $€ \mathfrak{F}, E_{n} \leqq A(n=1,2, \ldots)$ such that $\operatorname{Sup} m\left(E_{n}\right)=m_{*}(A)$. Without losing the generality we may assume that the sequence $\left\{E_{n}\right\}$ is ascending. Put $H=\bigcup_{n=1}^{\infty} E_{n}$, then we have, by $2^{\circ}, m\left(H^{a}\right)=\lim _{n \rightarrow \infty} m\left(E_{n}\right)$ $=m_{*}(A) . \quad H$ is open and $\leqq A$. If an open set $B \neq \mathfrak{O}$ is contained in $A-H$, then there exists, by $1^{\circ}$, a set $E \in \Subset$ with $E \leqq B m(E)>0$. Thus we have

$$
m\left(E_{n}+E\right)=m\left(E_{n}\right)+m(E)>m_{*}(A)
$$

for sufficiently large $n$, contrary to the definition of $m_{*}(A)$ and $E_{n}$ $+E \leqq A$.

Theorem 5. $m^{*}(G)=m_{*}(G)$ if $G$ is open.

Proof. There exists, by lemma 2, an open set $H \leqq G$ such that $m\left(H^{a}\right)=m_{*}(G)$ and $G-H$ does not contain open set $\neq \mathfrak{D}$. We have

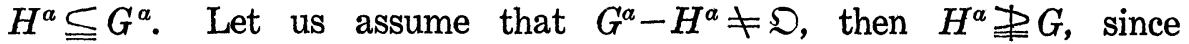
$H^{a} \supseteqq G$ implies the relation $G^{a} \leqq H^{a a}=H^{a}$ contrary to the assumption $G^{a}-H^{a} \neq \mathfrak{D}$. Thus the open set $G \cap H^{a c}$ is not void. This contradicts to the fact that $G-H$ does not contain open set $\neq \supseteq$. Therefore $H^{a}=G^{a}$ and thus we have, by lemma 2 and theorem 2

$$
m_{*}(G)=m\left(H^{a}\right)=m\left(G^{a}\right)=m^{*}(G)
$$

Theorem 6. The class $\Re$ of all sets $A$ such that $m^{*}(A)=m_{*}(A)$ is a countably additive class. Hence, by theorem $5, \Omega$ contains all Borel sets

Proof. We have to show (i) and (ii) : 


$$
\begin{aligned}
& A^{c} \in \Re \text { if } A \in \Re . \\
& \bigcup_{n=1}^{\infty} A_{n} \in \Re \quad \text { if } A_{n} \in \Re \quad(n=1,2, \ldots) .
\end{aligned}
$$

Proof of $(i)$. We see, by lemma 1, that $m^{*}(A)=m_{*}(A)$ implies $m_{*}\left(A^{c}\right)=1-m^{*}(A)=1-m_{*}(A)=m^{*}\left(A^{c}\right)$.

For the proof of (ii), we need the following two lemmas.

Lemma 3. If $m^{*}(A)=m_{*}(A)$, then we have $m^{*}\left(A^{a}-A^{i}\right)=0$ and hence $m^{*}\left(A-A^{i}\right)=0, m^{*}\left(A^{a}-A\right)=0$.

Proof. For every $\varepsilon>0$, there exists $E_{1} \in \mathfrak{E}, E_{2} \in \mathbb{E}$ such that $E_{1} \supseteqq A^{a}, E_{2} \leqq A^{i}$ and $m\left(E_{1}\right)<m^{*}\left(A^{a}\right)+\varepsilon, m\left(E_{2}\right)>m_{*}\left(A^{i}\right)-\varepsilon$. Hence we have $m^{*}\left(A^{a}-A^{i}\right) \leqq m\left(E_{1}-E_{2}\right)=\left(E_{1}\right)-m\left(E_{2}\right)<m^{*}\left(A^{a}\right)-m_{*}\left(A^{i}\right)+2 \varepsilon$ $=2 \varepsilon$, by theorem 2 and 3 .

Q.E. D.

Lemma 4. $m^{*}\left(\bigcup_{n-1}^{\infty} A_{n}\right)=0 \quad$ if $m^{*}\left(A_{n}\right)=0 \quad(n=1,2, \ldots)$.

Proof follows from the definition of $m^{*}$ and theorem 1 .

Proof of (ii) of theorem 6. We have

$$
\text { Q.E.D. }
$$

$$
m^{*}\left(\bigcup_{n=1}^{\infty} A_{n}\right)=m^{*}\left(\bigcup_{n=1}^{\infty} A_{n}^{i}+\bigcup_{n=1}^{\infty}\left(A_{n}-A_{n}^{i}\right)\right)
$$

By lemma 3 and $4, m^{*}\left(\bigcup_{n=1}^{\infty}\left(A_{n}-A_{n}^{i}\right)\right)=0$ and hence $m^{*}\left(\bigcup_{n=1}^{\infty} A_{n}\right)=m^{*}\left(\bigcup_{n=1}^{\infty} A_{n}^{i}\right)$ which is $=m_{*}\left(\cup A_{n}^{i}\right)$ by theorem 5. Therefore $m^{*}\left(\bigcup_{n=1}^{\infty} A_{n}\right) \leqq m^{*}\left(\bigcup_{n=1}^{\infty} A_{n}\right)$

Theorem 7. $m^{*}(A)=0$ implies that $A$ is non-dense, and conversely $m^{*}(A)=0$ if $A$ is non-dense.

Proof. We have, by theorem $2, m^{*}\left(A^{a}\right)=m^{*}(A)=0$. Accordingly $A^{a}$ does not contain open set $\neq \mathfrak{N}$, and so $A$ is non-dense. Next let $A$ be non-dense, then we have $A^{a i}=\mathfrak{D}$. Thus, by theorem 2,3 , and 6 $m^{*}(A)=m^{*}\left(A^{a}\right)=m_{*}\left(A^{a}\right)=m_{*}\left(A^{a i}\right)=m_{*}(\mathfrak{D})=0$.

Definition 3. $A$ set $A$ will be called measurable with respect to the outer measure $m^{*}$, if $m^{*}(B)=m^{*}(B \cap A)+m^{*}\left(B \cap A^{c}\right)$ for every set $B$.

Lemma 5. Let $A_{1}$ and $A_{2}$ be respectively contained in $G_{1}$ and $G_{2}$ where $G_{i}$ are mutually disjoint open sets, then

$$
m^{*}\left(A_{1}+A_{2}\right)=m^{*}\left(A_{1}\right)+m^{*}\left(A_{2}\right)
$$

Proof. $G_{1} \cap G_{2}=\mathfrak{D}$ implies $G_{1} \leqq G_{2}^{c}$. So we have $G_{1}^{a} \leqq G_{2}^{c a}=G^{c}$ and hence $G_{1}^{a} \cap G_{2}=\mathfrak{D}$. Since, by the assumption, the closure of an open set is open, we obtain $G_{1}^{a} \cap G_{2}^{a}=\mathfrak{D}$ by the same argument. Thus we may assume that $G_{1}$ and $G_{2}$ both $€ \mathbb{E}$ and hence $m^{*}\left(A_{1}+A_{2}\right)$ $=m^{*}\left(A_{1}\right)+m^{*}\left(A_{2}\right)$.

Theorem 8. If $m^{*}(A)=m_{*}(A)$ viz. $A \in \Re$, then $A$ is measurable with respect to $m^{*}$.

Proof. $m^{*}(B \cap A)+m^{*}\left(B \cap A^{c}\right)=m^{*}\left(B \cap A^{i}+B \cap\left(A-A^{i}\right)\right)+$ $m^{*}\left(B \cap A^{a c}+B \cap\left(A^{a}-A\right)\right)$. By lemma $3, B \cap\left(A-A^{i}\right)$ and $B \cap$ $\left(A^{a}-A\right)$ are of outer measure zero. Thus $m^{*}(B \cap A)+m^{*}\left(B \cap A^{c}\right)$ $=m^{*}\left(B \cap A^{i}\right)+m^{*}\left(B \cap A^{a c}\right)$. As $A^{i}$ and $A^{a c}$ are open we have, 
by lemma $5, m^{*}\left(B \cap A^{i}\right)+m^{*}\left(B \cap A^{a c}\right)=m^{*}\left(B \cap\left(A^{i}+A^{a c}\right)\right) \leqq m^{*}(B)$. Therefore, by theorem $2, m^{*}(B \cap A)+m^{*}\left(B \cap A^{c}\right)=m^{*}(B)$.

Theorem 9. If $A$ is measurable with respect to $m^{*}$, then $m^{*}(A)$ $=m^{*}(A)$ viz. $A \in \Re$,

Proof. We have $m^{*}(B)=m^{*}(B \cap A)+m^{*}\left(B \cap A^{c}\right)$ for any $B$. Hence, by putting $B=\Omega, 1=m^{*}(A)+m^{*}\left(A^{e}\right)$. Thus, by lemma 1 , we have $m^{*}(A)=m_{*}(A)$. in $\Omega$.

Theorem 10. The following conditions are mutually equivalent
(i) $A$ is non-dense.
(ii) $A$ is of first category.
(iii) $A$ is of measure zero.

Proof is obtained from theorem 7 and lemma 4.

Theorem 11. The following conditions are mutually equivalent in $\Omega$.

(i) $m^{*}(A)=m_{*}(A)$.

(ii) $A^{a i}=A^{i a}$.

(iii) $A$ is measurable with respect to $m^{*}$.

(iv) $A$ enjoys Baire's property viz. there exists an open set $G$ such that $A \cup G-A \cap G$ is of first category.

Proof. The implication (i) $\rightleftarrows$ (iii) is assured by theorem 8 and 9 . Since $A^{a i}=A^{a c a c}$, we have $A^{a i} \in \mathbb{E}$ and hence $A^{a i} \geqq A^{i a}$. $A^{i a}$ also $\in \mathbb{E}$ by the definition of $\mathbb{5}$. Thus if $m^{*}(A)=m_{*}(A)$, then $m\left(A^{a i}\right)=m_{*}\left(A^{a i}\right)$ $=m_{*}\left(A^{a}\right)=m^{*}\left(A^{a}\right)=m^{*}(A)=m_{*}(A)=m_{*}\left(A^{i}\right)=m^{*}\left(A^{i}\right)=m^{*}\left(A^{i a}\right)=$ $m\left(A^{i a}\right)$, and hence $A^{a i}=A^{i a}$ by $1^{\circ}$. Therefore (i) implies (ii). Conversely let $A^{a i}=A^{i a}$, then $m(A)=m^{*}\left(A^{\alpha}\right)=m_{*}\left(A^{a}\right)=m_{*}\left(A^{a i}\right)=m\left(A^{a i}\right)$ $=m\left(A^{i a}\right)=m^{*}\left(A^{i a}\right)=m^{*}\left(A^{i}\right)=m_{*}\left(A^{i}\right)=m_{*}(A)$, by theorem 2,3 and 6 . Hence (ii) implies (i). By theorem 10 and lemma 3, we see that (i) implies (iv). Since a set of first category is of measure zero by theorem 10, we see that (iv) implies (iii) and hence (i).

Corollary. The totality of measurable functions coincides with the totality of functions having Baires property.

Theorem 12. $m^{*}$ is an outer measure in the sense of Caratheodory.

Proof. $A \leqq B$ implies $m^{*}(A) \leqq m^{*}(B)$ by theorem 2. By theorem theorem 1 and the definition of $m^{*}$, we see that $m^{*}\left(\bigcup_{n=1}^{\infty} A_{n}\right) \leqq \sum_{n=1}^{\infty} m^{*}\left(A_{n}\right)$. Thus it will be sufficient to show that $m^{*}(A+B)=m^{*}(A)+m^{*}(B)$ if $A^{a} \cap B^{a}=\mathfrak{D}$. Being bicompact, $\Omega$ is normal and so there exist two

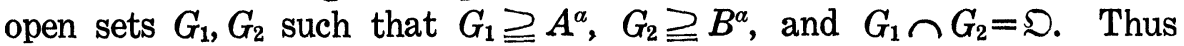
by lemma 5 , we have $m^{*}(A+B)=m^{*}(A)+m^{*}(B)$.

Theorem 13. For functions $f(x)$ defined on $\Omega$ the following conditions are mutually equivalent.

(i) $f(x)$ is a mesaurable function.

(ii) $f(x)$ is a function having Baire's property. 
(iii) There exists a continuous function (which may take the value $\left.\pm \infty^{11}\right)$, coinciding with $f(x)$ except on a set of measure zero.

(iv) The set of points of discontinuity of $f(x)$ is of measure zero.

Proof. The equivalence of (i) and (ii) is already proved in the corollary of theorem 11. The equivalence of (ii) and (iii) is proved by T. Ogasawara ${ }^{2}$. Next let there exist a continuous function $g(x)$ differing from $f(x)$ only on a set of measure zero. Thus there exists, for every $\varepsilon>0$, a set $E \in\left[\right.$ such that $m(E)<\varepsilon$ and $f(x)=g(x)$ on $E^{a}$. Hence $f(x)$ is continuous on the closed open set $E^{c}$. Since $\varepsilon$ was arbitrary, we obtain (iv). Conversely let the set $A$ of discontinuity of $f(x)$ satisfy $m^{*}(A)=0$, then $f(x)$ is continuous on $A^{c} \in \Re$. For any $\alpha$, the set $B=\{x \in \Omega ; f(x)>\alpha\}$ is $=\left\{x \in A^{c} ; f(x)>\alpha\right\}+\{x \in A ; f(x)>\alpha\}$. As $f(x)$ is continuous on $A^{c}$ we have $\{x \in A ; f(x)>\alpha\}=A^{c} \cap G$ with some open set $G$ of $\Omega$, and hence $A^{c} \cap G \in \Re$. Moreover $\{x \in A ; f(n)>\alpha\}$ is of measure zero with $A$. Therefore $B \in \Omega$, proving that (iv) implies (i).

1) If $f\left(x_{0}\right)= \pm \infty$, there exists, for every $a$ an open set $G_{a} \ni x_{0}$ such that $f(x) \gtrless a$ on $G_{a}$.

2) 日本數學物理學會誌, 16 兊, 9 號, 412 頁. 